\title{
MHD natural convection flow of Casson fluid in an annular microchannel containing porous medium with heat generation/absorption
}

https://doi.org/10.1515/nleng-2020-0010

Received Aug 3, 2019; accepted Mar 11, 2020.

\begin{abstract}
This study has been conducted to focus on natural convection flow of Casson fluid through an annular microchannel formed by two cylinders in the presence of magnetic field. The process of heat generation/absorption is taken into consideration. Combined effects of various parameters such as porous medium, velocity slip and temperature jump are considered. Solution of the present mathematical model is obtained numerically using fourthfifth order Runge-Kutta-Fehlberg method. The flow velocity, thermal field, skin friction and Nusselt number are scrutinized with respect to the involved parameters of interest such as fluid wall interaction parameter, rarefaction parameter, Casson parameter and Darcy number with the aid of graphs. It is established that higher values of Casson parameter increases the skin friction coefficient. Further it is obtained that rate of heat transfer diminishes as fluid wall interaction parameter increases.
\end{abstract}

Keywords: annular microchannel, Casson fluid, porous medium, fluid wall interaction, rarefaction

\section{Introduction}

Increase in trend towards micro scale system leads to analyze the fluid flow through micro passage such as micro heat exchangers, micro nozzles and microchannel etc. Micro scale system has tremendous application in the field of biomedicine, pharmaceutical chemistry, micro processing, engineering applications and electronics. Influence of Soret and Dufour effect on MHD flow of Casson fluid over

\footnotetext{
*Corresponding Author: B.J. Gireesha, Department of Studies and Research in Mathematics, Kuvempu University, Shankaraghatta-577451, Shivamogga, Karnataka, India, E-mail: bjgireesu@gmail.com

S. Sindhu, Department of Studies and Research in Mathematics, Kuvempu University, Shankaraghatta-577451, Shivamogga, Karnataka, India, E-mail: sindhusundars1@gmail.com
}

stretching sheet has been studied by Hayat et al. [1] using Homotopic method. They conclude that rise in values of Casson parameter and Hartmann number decreases the velocity. Heat and mass transfer analysis of Casson fluid over a stretching surface was conducted by Raju et al. [2]. A comparative study of Casson and Newtonian fluids is also done in this work. They obtained that enhancement in thermal boundary layer is achieved by increasing viscous dissipation. Mixed convection flow over a stretching surface of Casson fluid has been investigated numerically by Rana et al. [3] with homogeneous and heterogeneous heating. It is found that increase in Casson and slip parameter decreases the heat flux. Kumar et al. [4] examined the heat transfer of a Carreau fluid over a stretching sheet. They established that thickness of thermal boundary layer declines for large Prandtl number. Scrutinization of MHD Casson fluid flow past a wedge has been analyzed by Zaib et al. [5] considering activation energy and binary chemical reaction. It is found that velocity for both Newtonian and non-Newtonian fluid increases for increasing magnetic parameter. Influence of homogeneous and heterogeneous reactions on Casson fluid has been studied by Khan et al. [6]. It has been shown that temperature profile diminishes for higher values of Prandtl number. MHD flow of Casson fluid over a moving cylinder of linear velocity has been investigated by Tamoor et al. [7]. It is evident that skin friction coefficient diminishes for higher values of Casson parameter. Casson liquid flow induced over a sheet with nonlinear velocity has been studied by Khan et al. [8]. They obtained that concentration profile decrease for increasing values of homogeneous and heterogeneous parameter. Rheological Casson fluid model was employed in this study. Scrutinization of heat and mass characteristics of Casson and Williamson fluid has been discussed numerically by Kumaran and Sandeep [9] by considering Brownian and thermophoresis effect. It is concluded that impact of Lorentz force is higher on Williamson fluid than Casson fluid. Effect of viscous dissipation on MHD flow of Casson liquid over a vertical stretching plate has been studied by Gireesha et al. [10]. They found that for higher values of magnetic parameter, momentum boundary layer thick- 
ness diminishes. Kumar et al. [11] deliberated the mixed convection flow of Casson fluid over a vertical plate considering nonlinear radiation. It is shown that, temperature increases as Dufour effect and Soret parameter increases.

Analytical study of natural convection in a vertical microchannel has been carried out by Chen and Weng [12]. They conclude that volume flow rate at micro scale is higher compared to macro scale for natural convection in most situation. Impact of magnetic field on Casson liquid has been studied by Akbar et al. [13]. It is obtained that rise in values of magnetic parameter leads to increase of velocity profile. Impact of suction/injection on porous microchannel has been studied by Jha and Aina [14]. The result depicts that rate of heat transfer increases as mixed convection parameter increases. Jha et al. [15] investigated the natural convection flow in an annular microchannel analytically in the presence of velocity slip and temperature jump. It is found that increasing Knudsen number enhances the volume flow rate. Reddy et al. [16] investigated the entropy generation analysis of hydromagnetic Casson fluid over a cylinder. It is shown that increasing Casson parameter leads to enhancement in Bejan number. Convection flow of Casson fluid through a vertical channel has been studied by Khan et al. [17]. They employed Laplace method to describe velocity and temperature profiles. Pop et al. [18] numerically studied Casson fluid in a square cavity considering the combined effect of thermal radiation and viscous dissipation. The result shows that heat transfer rate enhances for increasing values of Casson parameter. Jha and Aina [19] investigated the influence of induced magnetic field on fully developed convection flow in an annular microchannel. It is obtained that for induced magnetic field, skin friction at the annular microchannel surface is higher.

Soret induced natural convection flow in an annular channel has been studied by Jha et al. [20]. They deliberated the heat and mass transfer analysis through channel. It is obtained that volume flow rate enhances with increasing mass transfer area. Sheremet and Pop [21] discussed the flow behavior of free convection in a triangular cavity filled with porous medium employing Bongiorno's model. It is evident that Sherwood number is an increasing function of Lewis number. Makinde et al. [22] explored the characteristics of heat and mass transfer through porous plate. MHD boundary flow is considered for this investigation. They studied the effect of temperature dependent viscosity on Nusselt number. Rate of heat transfer rises with temperature dependent viscosity. Mabood et al. [23] explored the MHD flow of nanofluid. Effect of chemical reaction, viscous dissipation and radiation are taken into account. It is obtained that skin friction coefficient increases as perme- ability parameter increases. Fully developed flow between two vertical cylinders were analysed by Jha and Yusuf [24] considering porous medium. The outcome of this study reveals that velocity is the function of Darcy number and annular gap. Dar and Elangovan [25], predicted heat and mass transfer of couple stress fluid flow through channel. It is obtained that velocity profile increases as couple stress parameter increases. MHD flow of Carreau fluid over a stretching sheet in the presence of homogeneous and heterogeneous reaction was investigated by Hayat et al. [26]. They concluded that thermal boundary layer enhances for increasing values of Biot number. Ree-Eyring fluid over a parallel plate has been studied by Ramesh and Eytoo [27] considering heat transfer and slip boundary conditions. They analysed the effect of porous medium and Ree-Eyring fluid parameter. It is clear that velocity is an increasing function of Ree-Eyring fluid parameter.

Two dimensional laminar flow of a incompressible fluid over a flat surface has been carried out by Chamkha and Issa [28]. They obtained that local Nusselt number is the function of Hartmann number and wall suction velocity. Natural convection flow of viscous incompressible fluid over a wavy surface has been studied by Molla et al. [29] using Finite Difference Method. Impact of heat generation/absorption on velocity boundary flow is observed. Finite Volume Method has been employed by Chamkha [30] to study the laminar convection flow of an electrically conducting fluid considering heat generation/absorption. It is concluded that average Nusselt number decreases in the presence of internal heat generation. Impact of heat generation/absorption on nanofluid flow over a permeable stretched surface has been examined by Alsaedi et al. [31]. Influence of heat generation and absorption on Casson fluid flow over a vertical cone and flat plate are studied by Mythili et al. [32]. They found that heat generation/absorption plays significant role in defining heat transfer through moving fluid. Heat generation/absorption fluid in an annular microchannel has been studied by Jha and Aina [33]. It is evident that heat generation parameter enhances the volume flow rate. Influence of sinusoidal heating in a porous cavity has been examined by Cheong et al. [34] using finite difference method. It is concluded that rate of heat transfer is higher for wavy cavity than square cavity. Gireesha et al. [35] numerically studied the influence of exponential heat source on Casson nano liquid flow over a stretching plate considering activation energy and chemical reaction. It is obtained that value of Nusselt number is higher for nonlinear radiative heat process compared to linear radiation effect.

Microfluidics has tremendous application in science and engineering technology. More specifically, electronic 
cooling systems micromixers and micro heat exchanger. Therefore, the present work is intended to explore the impact of Casson fluid flow in an annular microchannel filled with porous material. In this study heat generation/absorption characteristic of Casson fluid through microchannel is investigated. The variation in velocity and temperature profile for different parameters is examined and is displayed through graphs. The important parameters such as skin friction and Nusselt number are discussed.

\section{Mathematical analysis}

Natural convection flow of an incompressible Casson fluid in a vertical annular microchannel formed by two concentric cylinders with porous medium is considered. Let $X$ axis is parallel to gravitational field $g$ in opposite direction while $r$ axis is in the radial direction. The radius of inner cylinder is $r_{1}$ and that of outer cylinder is $r_{2}$. The outer surface of the inner cylinder is heated to a temperature $T_{1}$ in such a way that the surrounding fluid having temperature $T_{0}<T_{1}$. Here the inner surface of the outer cylinder is maintained at temperature $T_{0}$. Natural convection occurs due to this temperature difference.

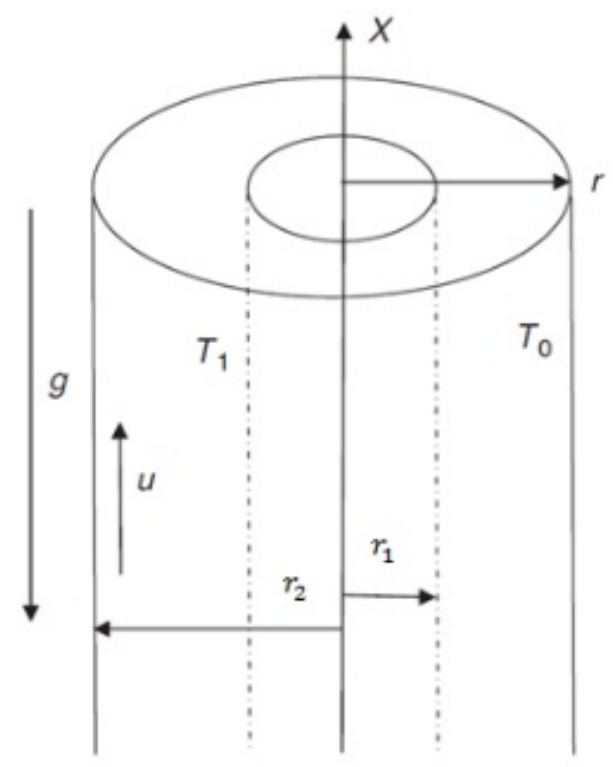

Figure 1: Geometry of the flow problem
Due to the flow is fully developed and cylinders are of infinite length, the transport phenomenon depends only on $r$. A uniform magnetic field of strength $B_{0}$ is applied normal to direction of flow. Heat generation/absorption process is considered in this analysis and is given by:

$$
Q=Q_{0}\left(T-T_{0}\right)
$$

Physical model of the present problem under Boussinesq approximation is given as follows;

Momentum equation:

$\frac{v}{r}\left[r\left(1+\frac{1}{\beta}\right) \frac{d^{2} u}{d r^{2}}+\frac{d u}{d r}\right]+g \beta_{0}\left(T-T_{0}\right)-\frac{v}{K} u-\frac{\sigma B_{0}^{2} u}{\rho_{0}}=0$

Energy equation:

$$
\frac{\alpha}{r} \frac{d}{d r}\left(r \frac{d T}{d r}\right) \pm \frac{Q}{\rho_{0} C_{p}}=0 .
$$

The boundary conditions for both velocity and temperature field are:

$$
\begin{gathered}
u=\left.\frac{2-\sigma_{v}}{\sigma_{v}} \lambda \frac{d u}{d r}\right|_{r=r_{1}} \text { at } r=r_{1}, \\
T=T_{1}+\left.\frac{2-\sigma_{t}}{\sigma_{t}} \frac{2 y}{y+1} \frac{\lambda}{P r} \frac{d T}{d r}\right|_{r=r_{1}} \text { at } r=r_{1}, \\
u=-\left.\frac{2-\sigma_{v}}{\sigma_{v}} \lambda \frac{d u}{d r}\right|_{r=r_{2}} \text { at } r=r_{2}, \\
T=T_{0}+\left.\frac{2-\sigma_{t}}{\sigma_{t}} \frac{2 y}{y+1} \frac{\lambda}{\operatorname{Pr}} \frac{d T}{d r}\right|_{r=r_{2}} \text { at } r=r_{2},
\end{gathered}
$$

On introducing following dimensionless quantities

$$
\begin{aligned}
& R=\frac{r-r_{1}}{w}, w=r_{2}-r_{1}, U=\frac{u}{u_{c}}, \theta=\frac{T-T_{0}}{T_{1}-T_{0}}, \\
& \eta=\frac{r_{1}}{r_{2}}, u_{c}=\frac{\rho_{0} g \beta_{0}\left(T_{1}-T_{0}\right)}{\mu_{0}} w^{2} .
\end{aligned}
$$

From equations (2)-(7), one can get

$$
\begin{aligned}
& \frac{1}{[\eta+(1-\eta) R]}\left(\left(1+\frac{1}{\beta}\right)[\eta+(1-\eta) R] \frac{d^{2} U}{d R^{2}}+(1-\eta) \frac{d U}{d R}\right) \\
& +\theta-\frac{U}{D a}-M^{2} U=0, \\
& \frac{1}{[\eta+(1-\eta) R]}\left([\eta+(1-\eta) R] \frac{d^{2} \theta}{d R^{2}}+(1-\eta) \frac{d \theta}{d R}\right) \pm S^{2} \theta=0 .
\end{aligned}
$$

Where, $\beta_{t}=\frac{2-\sigma_{t}}{\sigma_{t}} \frac{2 y}{y+1} \frac{1}{P r}, \quad \beta_{v}=\frac{2-\sigma_{v}}{\sigma_{v}}$ are dimensionless variables, $\lambda=\frac{\sqrt{\pi R T_{0 / 2 \mu_{0}}}}{\rho_{0}}$ is molecular free path, $S^{2}=\frac{Q_{0} w^{2}}{k_{0}}$ is heat generation/absorption parameter $M=\frac{\sigma B_{0}^{2} w^{2}}{\rho_{0} v}$ is Hartmann number, $D a=\frac{K}{w^{2}}$ is Darcy number, $\operatorname{Pr}=\frac{v}{\alpha}$ is 
Prandtl number, $K_{n}=\frac{\lambda}{w}$ is Knudsen number, $\ln =\frac{\beta_{t}}{\beta_{v}}$ is fluid wall interaction parameter and $\beta$ is Casson parameter. lows:

The corresponding boundary conditions are as fol-

$$
\begin{gathered}
U(0)=\left.\left(1+\frac{1}{\beta}\right) \beta_{v} K n \frac{d U}{d R}\right|_{R=0} \text { at } R=0 \\
\theta(0)=1+\left.\beta_{v} K n \ln \frac{d \theta}{d R}\right|_{R=0} \text { at } R=0 \\
U(1)=-\left.\left(1+\frac{1}{\beta}\right) \beta_{v} K n \frac{d U}{d R}\right|_{R=1} \text { at } R=1 \\
\theta(0)=-\left.\beta_{v} K n \ln \frac{d \theta}{d R}\right|_{R=1} \text { at } R=1 .
\end{gathered}
$$

Solution for energy equation is scrutinized considering two cases such as heat generation and absorption process. For heat generating case second term of equation (10) has positive sign. It has negative sign for heat absorption case.

\section{Case-I: Heat generation}

For this energy equation is as follows;

$$
\frac{1}{[\eta+(1-\eta) R]} \frac{d}{d R}\left([\eta+(1-\eta) R] \frac{d \theta}{d R}\right)+S^{2} \theta=0
$$

\section{Case-II: Heat absorption}

$$
\frac{1}{[\eta+(1-\eta) R]} \frac{d}{d R}\left([\eta+(1-\eta) R] \frac{d \theta}{d R}\right)-S^{2} \theta=0
$$

The two parameters of physical interest are rate of heat transfer/Nusselt number and skin friction at the cylinder surface are given by:

$$
\begin{gathered}
N u_{0}=-\left.\frac{d \theta}{d R}\right|_{R=0}, \\
N u_{1}=-\left.\frac{d \theta}{d R}\right|_{R=1}, \\
\tau_{0}=\left.\left(1+\frac{1}{\beta}\right) \frac{d U}{d R}\right|_{R=0}, \\
\tau_{1}=\left.\left(1+\frac{1}{\beta}\right) \frac{d U}{d R}\right|_{R=1} .
\end{gathered}
$$

Here, $N u_{0}, N u_{1}, \tau_{0}$ and $\tau_{1}$ are the Nusselt number and skin friction at the inner and outer cylinder surfaces respectively.

\section{Solution procedure}

The systems of ordinary differential equations (9)-(10) representing the defined flow problem along with the boundary conditions (11)-(14) are highly nonlinear. The boundary value problems are converted into initial value problem. The initial conditions are accessed with the help of shooting technique. Initially the equations are reduced to a system of first order simultaneous equations considering unknowns as follows:

$$
U=X_{1}, \frac{d U}{d R}=X_{2}, \quad \theta=X_{3}, \frac{d \theta}{d R}=X_{4},
$$

$\frac{d X_{2}}{d R}=\left(\frac{\beta}{1+\beta}\right)\left(X_{1}\left(\frac{1}{D a}+M^{2}\right)-\frac{1-\eta}{[\eta+(1-\eta) R]} X_{2}-X_{3}\right)$,

$\frac{d X_{4}}{d R}=-\left(S^{2} X_{3}+\frac{1-\eta}{[\eta+(1-\eta) R]} X_{4}\right)$ for heat absorption,

$\frac{d X_{4}}{d R}=S^{2} X_{3}-\frac{1-\eta}{[\eta+(1-\eta) R]} X_{4}$ for heat absorption.

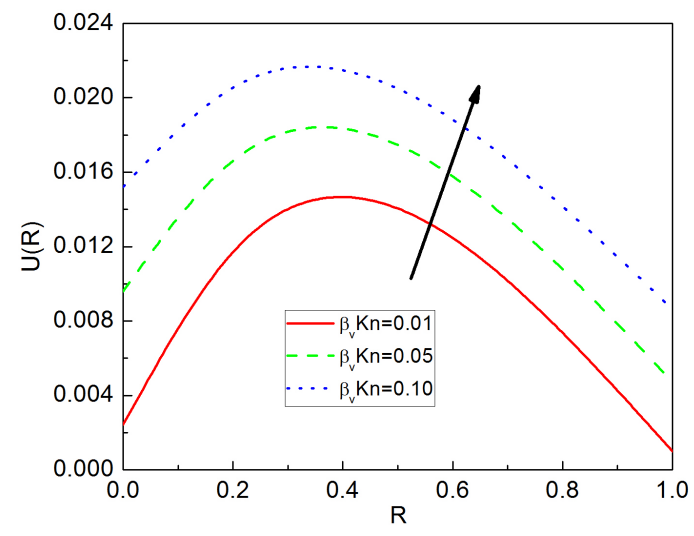

Figure 2: Impact of rarefaction parameter $\beta_{v} K_{n}$ on velocity profile

The corresponding boundary conditions (11)-(14) are reduced as below;

$$
\begin{gathered}
X_{1}(0)=\left(1+\frac{1}{\beta}\right) \beta_{v} K n \\
X_{2}(0), X_{2}(0)=m_{1},
\end{gathered}
$$




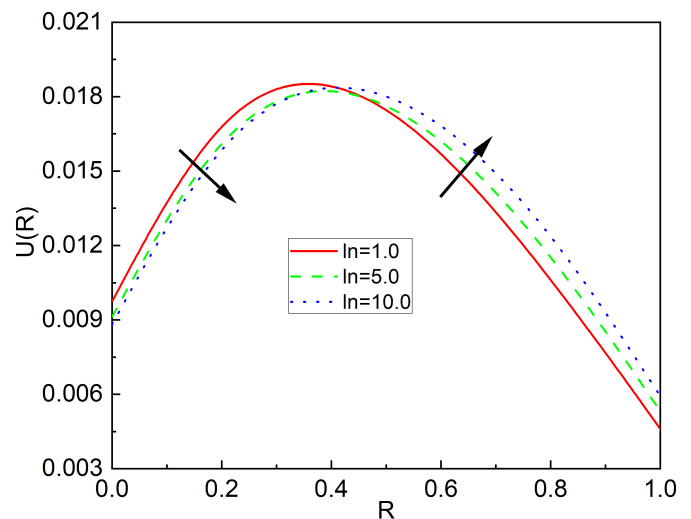

Figure 3: Impact of fluid wall interaction parameter $\ln$ on velocity profile

$$
\begin{gathered}
X_{3}(0)=1+\beta_{v} K n \ln X_{4}(0), \\
X_{4}(0)=m_{2} .
\end{gathered}
$$

These equations are solved by adopting Maple symbolic software which is capable of solving nonlinear ordinary differential equations using Runge Kutta Fehlberg fourth fifth order method.

\section{Results and discussion}

In this section we explored the nature of velocity profile, temperature profile, skin friction and Nusselt number for different flow parameters such as rarefaction parameter $\beta_{v} K n$, fluid wall interaction parameter $l n$, magnetic parameter $M$, heat generation/absorption parameter $S$, Darcy number $D a$ and Casson parameter $\beta$ with the help of graphical representation. The investigation is carried out for different values of $\beta_{v} K n=0.05, \ln =1.667, M=$ 2.0, $S=\beta=0.5, n=0.8$ and $D a=0.1$.

Figure 2 displays the effect of rarefaction parameter $\beta_{v} K n$ on velocity profile. As $\beta_{v} K n$ increases, we observe an increase in velocity slip at the surfaces, diminishes the retarding effect on the boundaries. This leads to enhancement in fluid velocity. It is obtained that velocity profile increases for rise in values of rarefaction parameter for both heat generation and absorption cases. Figure 3 exhibits the influence of fluid wall interaction parameter $\ln$ on velocity profile. It is shown that velocity profile decreases at inner cylinder surface whereas it increases at outer cylinder wall for both heat generation and absorption. Figure $4 \mathrm{a}$

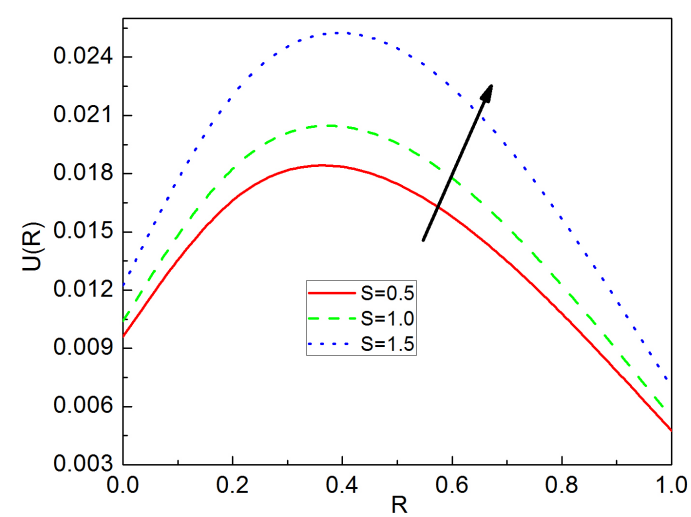

(a)

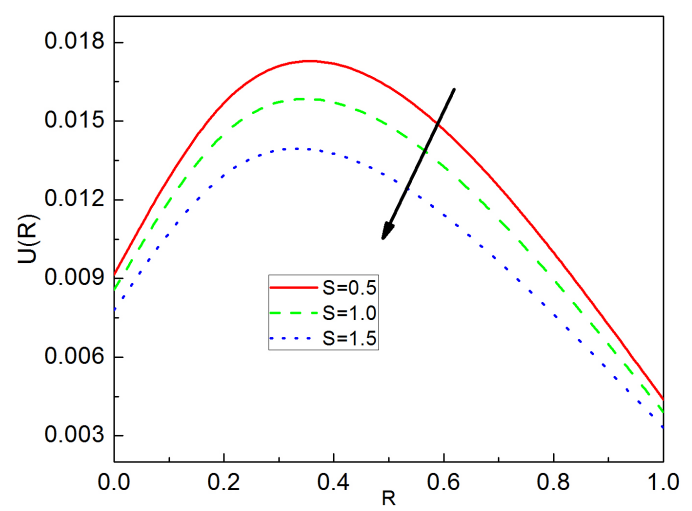

(b)

Figure 4: (a) (Case-I): Impact of heat generation/absorption parameter $S$ on velocity profile

(b)(Case-II): Impact of heat generation/absorption parameter $S$ on velocity profile

and $4 \mathrm{~b}$ predict the influence of heat generation/absorption parameter $S$ on velocity profile for heat generation and absorption cases respectively. From Figure $4 \mathrm{a}$ it is obtained that velocity profile enhances as the value of $S$ increases. Also, velocity retards with increasing values of heat absorption parameter. The impact of larger values of magnetic parameter $M$ on velocity profile is displayed in Figure 5. For larger magnetic parameter $M$, fluid velocity decelerates. This is due to the fact that the strong magnetic field develops Lorentz force (a resistive kind of force) which slows down the flow velocity.

Figures 6-9 represents the influence different parameters on enhancing heat transfer characteristics. The impact of increasing values of rarefaction parameter $\beta_{v} K n$ on 


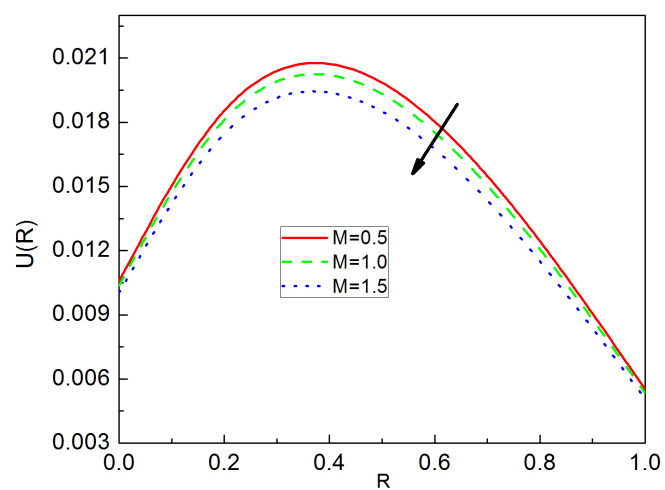

Figure 5: Impact of magnetic parameter $M$ on velocity profile

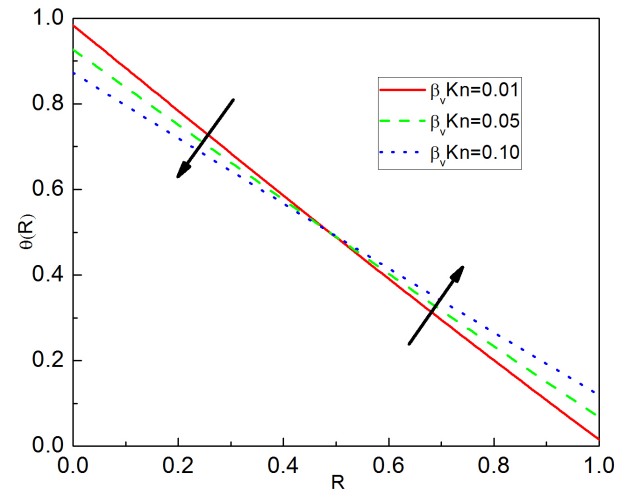

Figure 6: Impact of rarefaction parameter $\beta_{v} K n$ on temperature profile

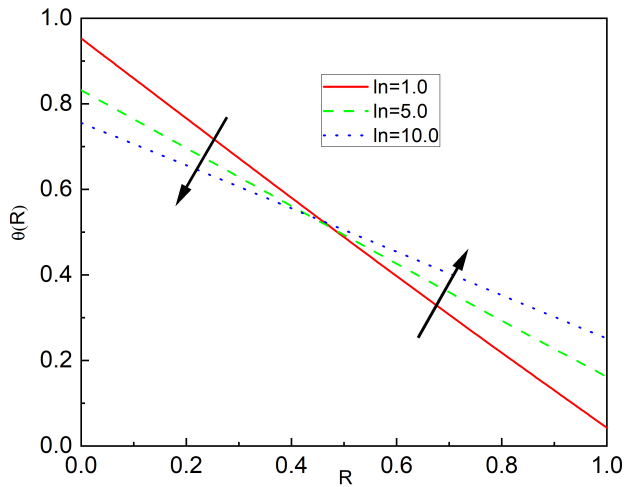

Figure 7: Impact of fluid wall interaction parameter $\ln$ on temperature profile

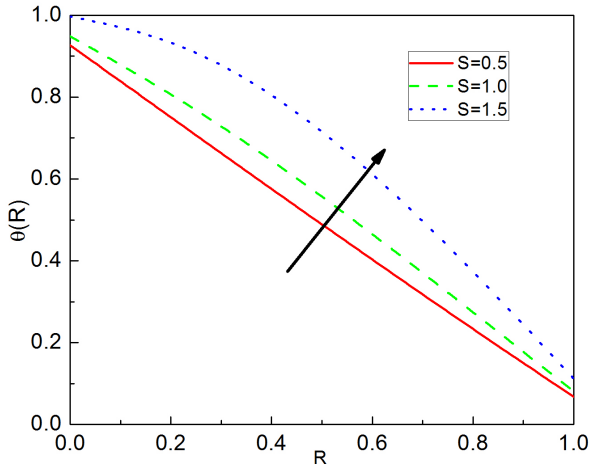

(a)

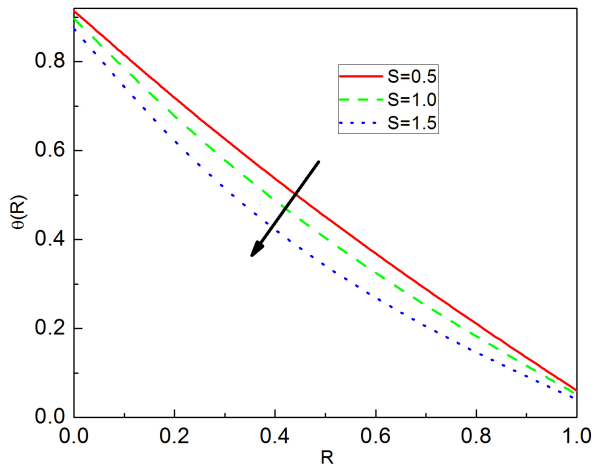

(b)

Figure 8: (a) (Case-I): Impact of heat generation/absorption parameter $S$ on temperature profile

(b)(Case-II): Impact of heat generation/absorption parameter $S$ on temperature profile

temperature profile is shown in Figure 6 for heat generation and absorption. Temperature profile shows decrease in trend as rarefaction parameter $\beta_{v} K n$ increases at inner cylinder. This is due to less interaction of fluid molecules and the hot wall of the cylinder. It is also observed that temperature enhances at outer cylinder. Figure 7 portrays the influence of fluid wall interaction parameter $\ln$ on temperature profile for both heat generation and absorption. It is observed that as fluid wall interaction parameter $\ln$ increases temperature decreases at the inner cylinder whereas it shows increase in trend towards outer cylinder surface. Figures $8 \mathrm{a}$ and $8 \mathrm{~b}$ interpret the effect of heat generation/absorption parameter $S$ on temperature profile for both heat generation and absorption respectively. Figure 8a shows that as heat generation/absorption parameter $S$ increases temperature also increases. This is because heat generation/absorption parameter $S$ warms the fluid. 
Hence an increment in the fluid temperature is observed. On rising values of heat generation/absorption parameter $S$ the fluid temperature decreases for heat absorption shown in Figure 8b.

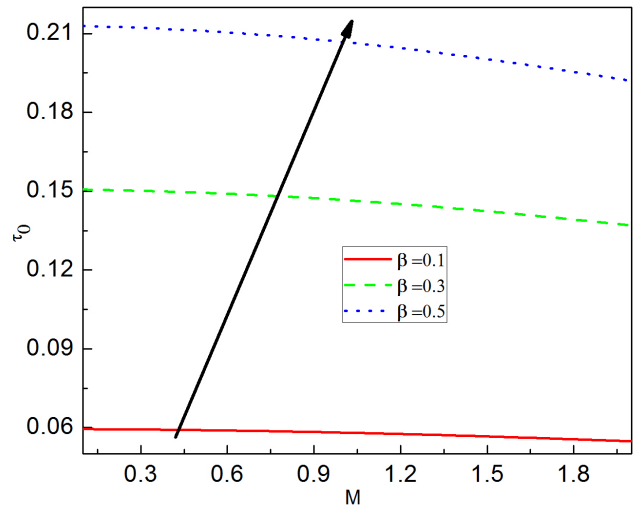

Figure 9: Impact of Casson parameter $\beta$ and magnetic parameter $M$ on skin friction at the inner cylinder

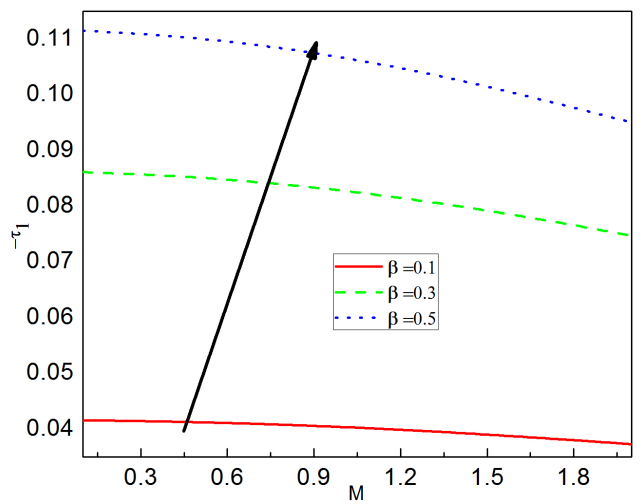

Figure 10: Impact of $\beta$ and $M$ on skin friction at the outer cylinder

Influence of Casson parameter $\beta$ and magnetic parameter $M$ on skin friction at the inner and outer cylinder is depicted in Figure 9 and 10 respectively. Higher value of Casson parameter enhances the skin friction coefficient. Variation of skin friction coefficient for increasing values of fluid wall interaction parameter and rarefaction parameter at inner cylinder $R=0$ and outer cylinder $R=1$ are shown in Figure 11 and Figure 12 respectively for both heat generation and absorption. For increasing values of fluid

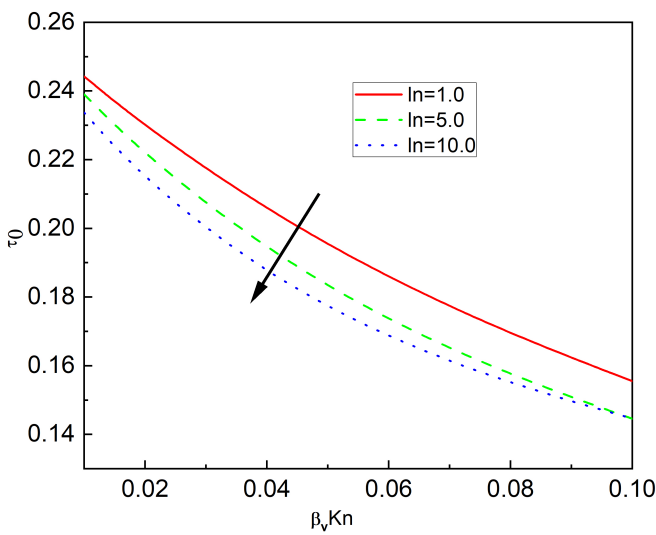

Figure 11: Impact of $\ln$ and $\beta_{v} K n$ on skin friction at the inner cylinder

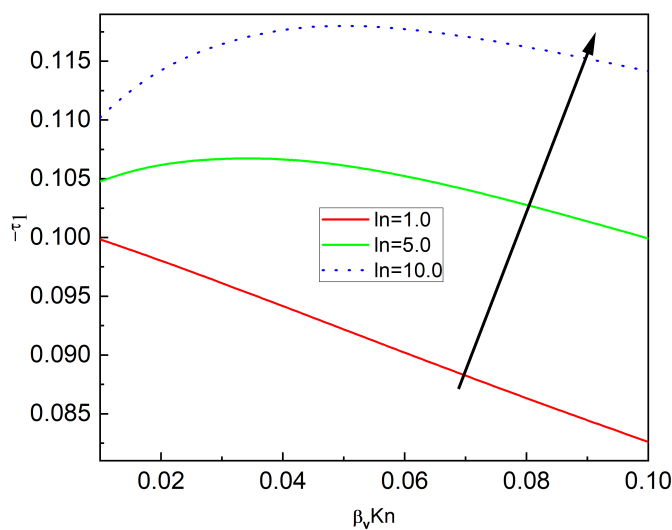

Figure 12: Impact of $\ln$ and $\beta_{v} K n$ on skin friction at the outer cylinder

wall interaction parameter $\ln$ from $1-10$, skin friction decreases at the outer surface of the inner cylinder is shown in Figure 11. It is observed from Figure 12 that there is an enhancement in skin friction coefficient for increasing values of fluid wall interaction parameter $\ln$ at the outer cylinder. Figure 13a exhibit the effects of heat generation parameter $S$ and rarefaction parameter $\beta_{v} K n$ on the skin friction at the inner cylinder. It is obtained that skin friction coefficient shows increase in trend for increasing values of heat generation parameter $S$. Impact of heat absorption parameter $S$ and rarefaction parameter $\beta_{v} K n$ at the inner cylinder is represented in Figure 13b, which shows decreasing trend for rising values of $S$. Figure 14a displays the effects of heat generation parameter $S$ and rarefaction parameter $\beta_{v} K n$ on skin friction coefficient at the inner surface of the outer cylinder $R=1$. It is obtained that skin friction coefficient 


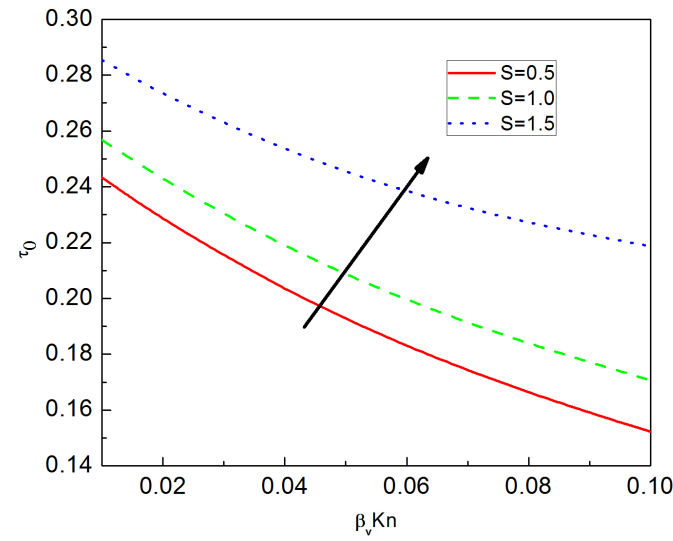

(a)

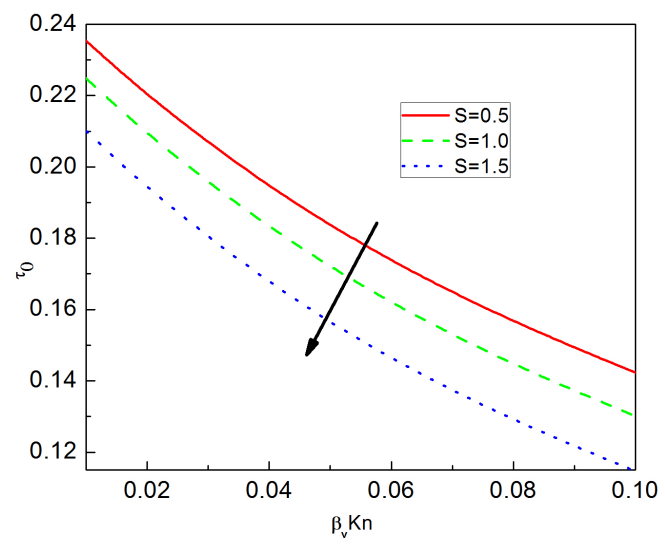

(b)

Figure 13: (a) (Case-I): Impact of $S$ and $\beta_{v} K n$ on skin friction at the inner cylinder

(b)(Case-II): Impact of $S$ and $\beta_{v} K n$ on skin friction at the inner cylinder

shows increase in trend for amassed values of heat generation parameter $S$. While it shows opposite trend in the case of heat absorption parameter $S$ which is represented in Figure 14b. Characteristics of Nusselt number $N u_{0}$ at the inner cylinder and Nusselt number $N u_{1}$ at the outer cylinder for varying values of fluid wall interaction parameter $l n$ and rarefaction parameter $\beta_{v} K n$ are represented graphically in Figure 15 and Figure 16 respectively. It is clear that rate of heat transfer diminishes for both heat generation and $\mathrm{ab}$ sorption at inner $(R=0)$ and outer cylinder $(R=1)$ surface for increasing values of $\ln$.

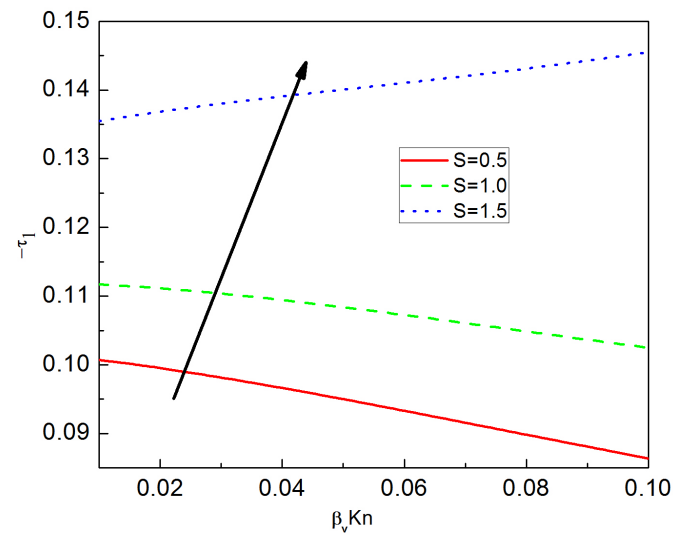

(a)

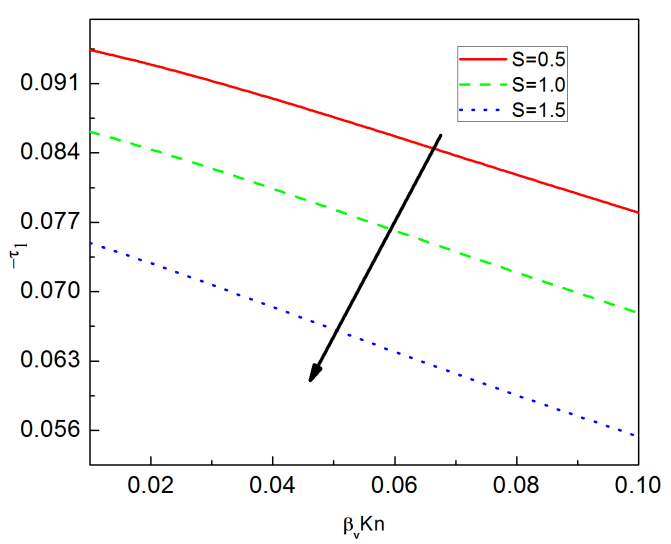

(b)

Figure 14: (a) (Case-I): Impact of $S$ and $\beta_{v} K n$ on skin friction at the outer cylinder

(b)(Case-II): Impact of $S$ and $\beta_{v} K n$ on skin friction at the outer cylinder

\section{Conclusions}

Fully developed steady state natural convection flow of Casson liquid through an annular microchannel filled with porous material is considered. The present investigation explores the impact of heat generation/absorption and applied magnetic field on Casson fluid flow in an annulus. Consequences of various flow parameters on flow velocity, temperature, Nusselt number and skin friction are discussed by means of graphs. The key outcome of the present analysis is as follows:

- Higher rarefaction parameter enhances the flow velocity of the Casson fluid. 


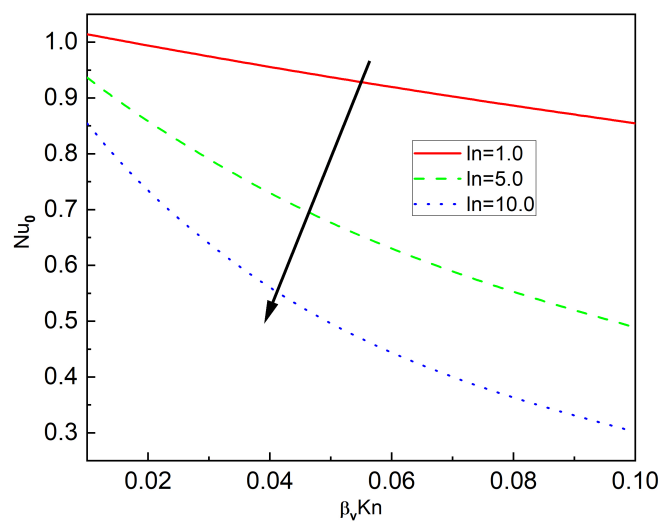

Figure 15: Impact of $\ln$ and $\beta_{v} K n$ on Nusselt number at the inner cylinder

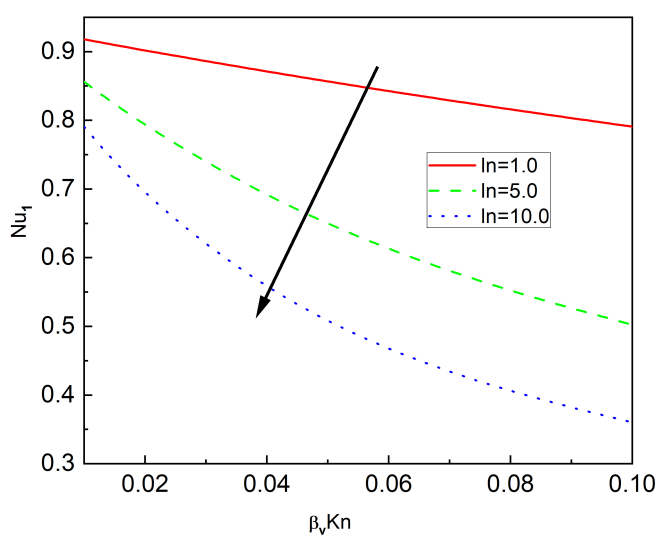

Figure 16: Impact of $\ln$ and $\beta_{v} K n$ on Nusselt number at the outer cylinder

- Magnetic parameter slowdowns the fluid flow through annular microchannel.

- Velocity and temperature profiles enhance for increasing values of heat generating parameter. Also, velocity and temperature profiles shows reverse trend in case of heat absorbing fluid.

- Casson parameter affects skin friction coefficient positively.

- Skin friction coefficient is an increasing function of heat generating parameter.

- Nusselt number diminishes for higher values of fluid wall interaction parameter $\ln$.

\section{Nomenclature:}

$w=$ channel width;

$P r=$ Prandtl number;

$T=$ Temperature;

$u=$ Velocity;

$C_{p}=$ specific heat at constant pressure;

$k=$ thermal conductivity;

$\beta_{t}, \beta_{v}=$ dimensionless variables;

$N u_{0}=$ Nusselt number at the inner cylinder;

$g=$ acceleration due to gravity;

$N u_{1}=$ Nusselt number at outer cylinder;

$l n=$ fluid wall interaction parameter;

$K n=$ Knudsen number;

$T_{0}=$ free stream temperature;

$M=$ Hartmann number;

$\tau_{0}=$ Skin friction at inner cylinder surface;

$\tau_{1}=$ Skin friction at outer cylinder surface;

and $Q_{0}, r=$ radial coordinate.

\section{Greek symbols:}

$\alpha=$ thermal diffusivity;

$\beta=$ Casson parameter;

$\sigma=$ Electrical conductivity;

$\sigma_{v}=$ thermal momentum accommodation coefficient;

$\sigma_{t}=$ tangential momentum accommodation coefficient;

$\lambda=$ molecular mean free path;

$v_{0}=$ fluid kinematic viscosity;

$\mu_{0}=$ dynamic viscosity;

$\rho_{0}=$ density;

$\theta=$ dimensionless temperature.

\section{References}

[1] Hayat T., Shehzad S.A., Alsaedi A., Soret and Dufour effects on magnetohydrodynamic (MHD) flow of Casson fluid, Appl. Math. Mech., 2012, 33, 1301-1312.

[2] Raju C.S.K., Sandeep N., Sugunamma V., Babu M.J., Reddy J.V.R., Heat and mass transfer in magnetohydrodynamic Casson fluid over an exponentially permeable stretching surface, Eng. Sci. Technol. Int. J., 2016, 19, 45-52.

[3] Rana S., Mehmood R., Akbar N.S., Mixed convective oblique flow of a Casson fluid with partial slip, internal heating and homogeneous-Heterogeneous reaction, J. Mol. Liq., 2016, 222, 1010-1019.

[4] Ganesh Kumar K., Gireesha B.J., Rudraswamy N.G., Manjunatha S., Radiative heat transfers of Carreau fluid flow over a stretching sheet with fluid particle suspension and temperature jump, Res. Phys., 2017, 7, 3976-3983.

[5] Zaib A., Rashidi M.M., Chamkha A., Bhattacharyya K., Numerical solution of second law analysis for MHD Casson nanofluid past a wedge with activation energy and binary chemical reaction, Int. J. Numer. Meth. Heat Fluid Flow, 2017, 27, 2816-2834. 
[6] Khan, M.I., Waqas, M., Hayat, T., Alsaedi, A., Colloidal study of Casson fluid with homogeneous-heterogeneous reactions, J. Colloid Interf. Sci., 2017, 498, 85-90.

[7] Tamoor M., Waqas M., Khan M.I., Alsaedi A., Hayat T., Magnetohydrodynamic flow of Casson fluid over a stretching cylinder, Res. Phys., 2017, 7, 498-502.

[8] Khan M.I., Hayat T., Khan M.I., Alsaedi A., A modified homogeneous-heterogeneous reactions for MHD stagnation flow with viscous dissipation and Joule heating, Int. J. Heat Mass Transf., 2017, 113, 310-317.

[9] Kumaran G., Sandeep N., Thermophoresis and Brownian moment effects on parabolic flow of MHD Casson and Williamson fluids with cross diffusion, J. Mol. Liq., 2017, 233, 262-269.

[10] Gireesha B.J., Ganesh Kumar K., Krishnamurthy M.R., Manjunatha S., Rudraswamy N.G., Impact of ohmic heating on MHD mixed convection flow of Casson fluid by considering Cross diffusion effect, Nonlin. Eng., 2018, 8, 380-388.

[11] Ganesh Kumar K., Archana M., Gireesha B.J., Krishanamurthy M.R., Rudraswamy N.G., Cross diffusion effect on MHD mixed convection flow of nonlinear radiative heat and mass transfer of Casson fluid over a vertical plate, Res. Phys., 2018, 8, 694701.

[12] Chen C.K., Weng H.C., Natural convection in a vertical microchannel, J. Heat Transf., 2005, 127, 1053-1056.

[13] Akbar N.S., Influence of magnetic field on peristaltic flow of a Casson fluid in an asymmetric channel: Application in crude oil refinement, J. Magn. Magn. Mater., 2015, 378, 463-468.

[14] Jha, B.K., Aina, B., Role of suction/injection on steady fully developed mixed convection flow in a vertical parallel plate microchannel, Ain Shams Engineering J, 2016, 9, 747-755.

[15] Jha B.K., Aina B., Rilwanu Z., Steady fully developed natural convection flow in a vertical annular microchannel having temperature dependent viscosity: An exact solution, Alexandria Eng. J., 2016, 55, 951-958.

[16] Reddy G.J., Kethireddy B., Kumar M., Hoque M.M., A molecular dynamics study on transient non-Newtonian MHD Casson fluid flow dispersion over a radiative vertical cylinder with entropy heat generation, J. Mol. Liq., 2017, 252, 245-262.

[17] Khan I., Saqib M., Ali F., Application of time-fractional derivatives with non-singular kernel to the generalized convective flow of Casson fluid in a microchannel with constant walls temperature, Europ. Phys. J. Special Topics, 2017, 226, 37913802.

[18] Pop I., Sheremet M., Free convection in a square cavity filled with a Casson fluid under the effects of thermal radiation and viscous dissipation, Int. J. Numer. Meth. Heat Fluid Flow, 2017, 27(10) , 2318-2332.

[19] Jha B.K., Aina B., Impact of induced magnetic field on magnetohydrodynamic (MHD) natural convection flow in a vertical annular micro-channel in the presence of radial magnetic field, Propul. Power Res., 2018, 7, 171-181.

[20] Jha B.K., Joseph S.B., Ajibade A.O., Role of thermal diffusion on double-diffusive natural convection in a vertical annular porous medium, Ain Shams Eng. J., 2015, 6, 29-637.

[21] Sheremet M.A., Pop I., Free convection in a triangular cavity filled with a porous medium saturated by a nanofluid, Int. J. Numer. Meth. Heat Fluid Flow, 2015, 25, 1138-1161.

[22] Makinde O.D., Khan W.A., Culham J.R., MHD variable viscosity reacting flow over a convectively heated plate in a porous medium with thermophoresis and radiative heat transfer,
International J. Heat Mass Transf., 2016, 93, 595-604.

[23] Mabood F., Shateyi S., Rashidi M.M., Momoniat, E., Freidoonimehr, N., MHD stagnation point flow heat and mass transfer of nanofluids in porous medium with radiation, viscous dissipation and chemical reaction, Adv. Powder Technol., 2016, 27, 742-749.

[24] Jha B.K., Yusuf T.S., Transient free convective flow in an annular porous medium: A semi-analytical approach, Eng. Sci. Technol. Int. J., 2016, 19, 1936-1948.

[25] Dar A.A., Elangovan K., Influence of an inclined magnetic field on Heat and Mass transfer of the peristaltic flow of a couple stress fluid in an inclined channel, World J. Eng., 2017, 14, 7-18.

[26] Hayat T., Khan M.I., Waqas M., Alsaedi A., Mathematical modeling of non-Newtonian fluid with chemical aspects: A new formulation and results by numerical technique, Colloids Surf. A: Physicochem. Eng. Asp., 2017, 518, 263-272.

[27] Ramesh K., Eytoo S.A., Effects of thermal radiation and magnetohydrodynamics on Ree-Eyring fluid flows through porous medium with slip boundary conditions, Multidisc. Model.Mater. Struct., 2019, 15(2), 492-507.

[28] Chamkha A.J., Issa C., Effects of heat generation/absorption and thermophoresis on hydromagnetic flow with heat and mass transfer over a flat surface, Int. J. Numer. Meth. Heat \& Fluid Flow, 2000, 10, 432-449.

[29] Molla M.M., Hossain M.A., Yao L.S., Natural convection flow along a vertical wavy surface with uniform surface temperature in presence of heat generation/absorption, Int. J. Therm. Sci., 2000, 43, 157-163.

[30] Chamkha A.J., Hydromagnetic combined convection flow in a vertical lid-driven cavity with internal heat generation or absorption, Numer. Heat Transf., Part A: Appl., 2012, 41, 529546.

[31] Alsaedi A., Awais M., Hayat T., Effects of heat generation/absorption on stagnation point flow of nanofluid over a surface with convective boundary conditions, Commun. Nonlin. Sci. Numer. Simul., 2012, 17, 4210-4223.

[32] Mythili D., Sivaraj R., Rashidi M.M., Heat generating/absorbing and chemically reacting Casson fluid flow over a vertical cone and flat plate saturated with non-Darcy porous medium, Int J. Numer. Meth. Heat Fluid Flow, 2017, 27, 156-173.

[33] Jha B.K., Aina B., Investigation of heat generation/absorption on natural convection flow in a vertical annular micro-channel: An exact solution, Multidisc. Model. Mater. Struct., 2017, 14, 143-167.

[34] Cheong H.T., Sivasankaran S., Bhuvaneswari M., Natural convection in a wavy porous cavity with sinusoidal heating and internal heat generation, Int J. Numer. Meth. Heat Fluid Flow, 2017, 27, 287-309.

[35] Gireesha B.J., Archana M., Mahanthesh B., Prasannakumara B.C., Exploration of activation energy and binary chemical reaction effects on nano Casson fluid flow with thermal and exponential space-based heat source, Multidisc. Model. Mater. Struct., 2018, 15, 227-245. 